\title{
Aspects of the Terrestrial ELF Noise Spectrum When Near the Source or Its Antipode
}

\author{
L. G. Abraham, Jr. \\ Applied Research Laboratory, Sylvania Electronic Systems, a Division of Sylvania Electric \\ Products, Inc., Waltham, Mass. 02154
}

(Received September 1, 1964; revised December 21, 1964)

\begin{abstract}
Radiowave propagation in the extremely low frequency (ELF) range of $3 \mathrm{~Hz}$ to $3 \mathrm{kHz}$ has been successfully treated in the past by a type of waveguide mode theory. This theory simplifies at large distances where only the zero order mode need be considered. It is shown in this paper that for frequencies in the band of $5 \mathrm{~Hz}$ to $1 \mathrm{kHz}$ the zero order mode predominates at any distance in excess of roughly $500 \mathrm{~km}$.

In a lower part of the ELF band $(5 \mathrm{~Hz}$ to $100 \mathrm{~Hz})$ more than one approximating form must be used. The commonly used asymptotic expansion applies only to a middle range of distances between source and receiver. When the receiver is within roughly one-sixth wavelength of either the source or its antipode then different approximations must be employed. These extensions of the theory are used in this paper to derive modifications of previous results for the terrestrial ELF noise power spectrum.
\end{abstract}

\section{Introduction}

The theory of extremely low frequency (ELF, $3 \mathrm{~Hz}$ to $3 \mathrm{kHz}$ ) radiowave propagation, developed by Schumann [1957] and Wait [1960a, b], predicts that the spherical cavity between the earth and the ionosphere will resonate at various frequencies for which the phase retardation in one circuit around the earth equals an integer number of complete cycles. This resonance phenomenon was observed experimentally in the natural noise background by Balser and Wagner [1960] over the "resonant" range of frequencies $(5$ to $50 \mathrm{~Hz}$ ). These observations were fitted in some detail by Raemer [1961] who derived an expression for the power spectrum of the lightning flashes thought to be responsible. Improved explanations were given by Galejs [1961a, 1962] who had previously developed a theory of ELF propagation $(5 \mathrm{~Hz}$ to $1 \mathrm{kHz}$ ) for a more realistic model of exponentially increasing conductivity with height in the isotropic ionosphere [Galejs, 196lb]. The earth's magnetic field was mostly ignored in these references and this paper will follow suit. The effect of such a static magnetic field has already been shown by Wait [1960b] to be largely negligible in the ELF band.

Some additions to the existing theory are needed in the resonance range when the noise source is close to the observer or his antipode. The work of both Raemer and Galejs was compared to observations taken by Balser and Wagner [1960, 1962] in the vicinity of Boston, Mass. The lightning stroke noise sources in these cases were mostly far distant from the receiver or its antipode. Hence, in this sense, the results of Raemer and Galejs are correct as they stand. The point of this paper is that they cannot be translated directly to measurements performed at sites close to the great tropical thunderstorm areas such as the Amazon or the Congo River basins (or their antipodes). All of the work presented here applies to the frequency range of $5 \mathrm{~Hz}$ to $1 \mathrm{kHz}$ in agreement with the work of Galejs [1961b].

The waveguide mode theory of radio propagation is useful at ELF [Wait, 1960b] where the earth and the ionosphere form the lower and upper boundaries, respectively. The distance along the earth's surface is measured by the angle $\theta$ subtended at its center. The individual modes in such a spherical system have a dependence on distance given by $P_{\nu(n)}(-\cos \theta)$, the Legendre function of argument $(-\cos \theta)$ and of complex degree $\nu(n)$. In this paper the zero order mode $(n=0)$ is defined to be the mode of least attenuation in the ELF band. It is commonly stated that at large distances the zero order mode will predominate and the others can be ignored. This paper shows 
that the critical distance at which this first occurs is roughly equal to $500 \mathrm{~km}$; a value large compared to the height of the ionosphere and, to the first order, constant with frequency in the range of $5 \mathrm{~Hz}$ to $1 \mathrm{kHz}$. The mode cutoff point around $3 \mathrm{kHz}$ is not treated.

This last statement may seem to be at variance with previous ideas that one-sixth wavelength is a minimum distance. What Wait [1960b] has shown is that the normalized magnitude of the electric field varies in a linear manner with distance when the distance exceeds one-sixth wavelength and the frequency is in the range of 50 to $1600 \mathrm{~Hz}$. However, the fact is that one-sixth wavelength also arises very naturally as the minimum distance needed to use a certain simplifying asymptotic expansion for $P_{\nu(0)}(-\cos \theta)$. For frequencies below $100 \mathrm{~Hz}$ there is a range of distances running from $500 \mathrm{~km}$ out to one-sixth wavelength where this first asymptotic form does not hold. But the zero order mode still predominates. Fortunately, a second expansion is available that does hold. Similarly, a third approximate form must be used when the radio wave source is within one-sixth wavelength of the observers' antipode. A second result of this paper is to present, under these latter two conditions, the necessary modifications to the formula of Galejs [1961a] for the terrestrial noise power spectrum.

\section{Background Results}

In support of the main points of this paper, it is useful to set down some previously developed approximations for the Legendre function $P_{\nu}(-\cos \theta)$ and the associated Legendre function of: first order $P_{\nu}^{1}(-\cos \theta)$. An approximation for the complex degree $\nu(n)$ as a function of $n(=1,2, \ldots)$ is also given. These are results that are needed in various parts of this paper.

\subsection{Approximate Forms for $P_{\nu}(-\cos \theta)$ and $P \nu^{1}(-\cos \theta)$}

The various mathematical approximations quoted here can be found either in Bateman Manuscript Project [1953] or Magnus and Oberhettinger [1949]. They are grouped according to the relative position of the noise source and the receiver. Further approximations are shown that apply more particularly for the zero order mode since the complex degree $\nu(0)$ is largely real [Galejs, 1961b].

The asymptotic expansions conventionally used in ELF propagation theory apply when the receiver is not too close to the source or its antipode. More explicitly,

$$
\begin{aligned}
& P_{\nu}(-\cos \theta) \simeq \frac{\Gamma(\nu+1)}{\Gamma(\nu+(3 / 2))} \sqrt{\frac{2}{\pi \sin \theta}} \cos [(\nu+(1 / 2))(\pi-\theta)-(\pi / 4)] \\
& P_{\nu}^{1}(-\cos \theta) \simeq \frac{\Gamma(\nu+2)}{\Gamma(\nu+(3 / 2))} \sqrt{\frac{2}{\pi \sin \theta}} \cos [(\nu+(1 / 2))(\pi-\theta)+(\pi / 4)]
\end{aligned}
$$

if $|\nu| \theta \gg 1$ and $|\nu|(\pi-\theta) \gg 1$ and

where $\Gamma(z)$ is the gamma function of $z$.

Each of these is the first term of a trigonometric expansion. If the Sterling formula is used to approximate the gamma functions, the asymptotic expansions reduce to

$$
\begin{aligned}
& P_{\nu}(-\cos \theta) \simeq \sqrt{2 /(\nu+(1 / 2)) \pi \sin \theta} \cos [(\nu+(1 / 2))(\pi-\theta)-(\pi / 4)] \\
& P_{\nu}^{1}(-\cos \theta) \simeq \sqrt{2(\nu+(1 / 2)) / \pi \sin \theta} \cos [(\nu+(1 / 2))(\pi-\theta)+(\pi / 4)]
\end{aligned}
$$

if $|\nu| \theta \gg 1$ and $|\nu|(\pi-\theta) \gg 1$. 
These last approximations are most appropriate in the case of the zero order mode where $\nu(0)$ is largely real.

When the source is close to the antipode of the receiver an expansion of $P_{\nu}(-\cos \theta)$ for small $(\pi-\theta)$ is required. Probably the most rapidly convergent is a series, due to MacDonald, in terms of Bessel functions [Bateman Manuscript Project, 1953]. However, for the computations in this paper a better choice is based on the hypergeometric series definition of the Legendre function,

$$
\begin{aligned}
& P_{\nu}(-\cos \theta) \simeq 1 \\
& P_{\nu}^{1}(-\cos \theta) \simeq-\nu(\nu+1) \cos (\theta / 2) \\
& \text { if }|\nu|(\pi-\theta) \ll 1 .
\end{aligned}
$$

Finally, when the sources are near the receiver itself a third approximation must be used to properly account for the singularity there. Materially different appearing expressions apply, depending on whether the order is zero or positve [Bateman Manuscript Project, 1953]:

$$
\begin{aligned}
& P_{\nu}(-\cos \theta) \simeq(\sin (\nu \pi) / \pi)[2 \log (\sin (\theta / 2))+C+2 \psi(\nu+1)+\pi \cot (\nu \pi)] \\
& P_{\nu}^{1}(-\cos \theta) \simeq-(\sin (\nu \pi) / \pi) / \sin (\theta / 2) \\
& \text { if }|\nu| \theta \ll 1 \text { and }
\end{aligned}
$$

where $C=$ Euler's constant $=0.5772-$

$\psi(z)=$ logarithmic derivative of the Gamma function

$$
=d(\log \Gamma(z)) / d z=-C+\sum_{n=0}^{\infty}[(1 /(n+1))-(1 /(n+z))] .
$$

These last results complete the approximations needed to cover the entire range of polar angle.

\subsection{Higher Order Roots of the Modal Equation}

Wait [1960a] has already shown in the ELF band for a homogeneous ionospheric layer that only the zero order mode is freely propagating. The higher order modes $(n \geqslant 1)$ are below cutoff and thus heavily attenuated with distance. The same result can be obtained, using the zero order mode solution by Galejs [1961b], for a more realistic, exponentially increasing conductivity of the ionosphere. (The exponential model had been previously treated by Wait [1960a, b]. The solution, due to Galejs, represents an extension to that work.)

The complex degree $\nu(n)$ is related to a mode factor $S_{n}$ by the relation

$$
\nu(\nu+1)=\left(k a S_{n}\right)^{2}
$$

where

$$
\begin{aligned}
& k=\text { free space, plane wave propagation constant } \\
& a=\text { radius of the earth. }
\end{aligned}
$$

The so-called modal equation is written in terms of $S_{n}$ to satisfy boundary conditions at the earth and at the ionosphere. In the ELF band Galejs showed for the exponential ionosphere that $S_{0}$ is largely real and close to unit magnitude, i.e., $\operatorname{Re}\left(S_{o}\right)>10 \operatorname{Im}\left(S_{0}\right)$ and $S_{0} \simeq 1$ for frequencies from 
$5 \mathrm{~Hz}$ to $1 \mathrm{kHz}$. He observed that this solution can be used to define an equivalent homogeneous ionosphere that will have an identical surface impedance $Z_{i}$.

It can be said that $S_{n}$ is a function of $Z_{i}$ which, in turn, is related to $S_{0}$ which is already known. Using the basic relations of $\mathrm{W}$ ait the end result is

$$
S_{n}^{2}=1-(\pi n / 2 k h)^{2}\left[1+\sqrt{1+(2 k h / \pi n)^{2}\left(1-S_{0}^{2}\right)}\right]^{2}
$$

where $h=$ height of the ionosphere above the earth. Detailed examination of numerical values for $S_{0}$ [Galejs, 1961b, 1962] shows that the square root can be expanded with little error. The parameter $(2 k h / \pi n)^{2}\left(1-S_{0}^{2}\right)$ has a maximum value of 0.1 at the upper frequency limit of $1 \mathrm{kHz}$.

After performing the expansion the higher order factors are approximated by

$$
S_{n}^{2} \simeq-(\pi n / k h)^{2}+\left(2 S_{0}^{2}-1\right)
$$

Solving for the higher order complex degree (5) shows that to a close approximation

$$
\nu(n) \simeq-j(\pi a n / h)+j\left(k^{2} a h / 2 \pi n\right)\left(2 S_{0}^{2}-1\right) \simeq-j(\pi a n / h) .
$$

In this form, the first constant term on the right predominates (as shown) for frequencies from $5 \mathrm{~Hz}$ to $1 \mathrm{kHz}$. The higher order modes $(n \geqslant 1)$ have a complex degree that is largely imaginary. Thus, it follows that they are rapidly attenuated with distance.

\section{Critical Distances}

The previous results are used in this section to help prove that the zero order mode begins to predominate at distances in excess of $500 \mathrm{~km}$. Also, the ranges of polar angle are specified where various approximate forms for this zero order mode apply.

\subsection{Minimum Distance for Predominance of the Zero Order Mode}

The vertical electric field at the earth's surface for a vertical current element at the surface is given by [Wait, 1960a,b],

$$
E_{r}=\left(\eta I d s / 2 k h a^{2}\right)\left\{\begin{array}{l}
\nu(0)(\nu(0)+1) P_{\nu(0)}(-\cos \theta) / 2 \sin \nu(0) \pi \\
+\sum_{n=1}^{\infty} \nu(\nu+1) P_{\nu}(-\cos \theta) / \sin \nu \pi
\end{array}\right\}
$$

where $\theta=$ polar angle subtended by the source and the observer at the center of the earth,

$\eta=$ characteristic impedance of free space $\simeq 120 \pi \Omega$,

$k=$ free space, plane wave propagation constant,

$I d s=$ moment of the vertical element,

$a=$ radius of the earth,

$h=$ height of the ionosphere above the earth.

Near the source all of the modes are required. At larger distances only the zero order mode has any significance since all the higher order modes are below cutoff.

The cutoff effect can be clearly seen by the following approximation which applies at larger distances for the higher order mode term in the series for electric field.

$$
\left|\nu(\nu+1) P_{\nu}(-\cos \theta) / \sin \nu \pi\right| \simeq(\pi a n / h)^{3 / 2} \sqrt{2 / \pi \sin \theta} \exp (-(\pi a n / h) \theta)
$$


for $n \geqslant 1$

$$
\text { if }|\nu| \theta \gg 1 \text { and }|\nu|(\pi-\theta) \gg 1 \text {. }
$$

(This result uses those of (2), (5), (7), and (8).) The parameter ( $\pi$ an/h) has a minimum value of about 200 for an ionosphere of maximum height, $h=100 \mathrm{~km}$. Hence, the exponential reduces the amplitude to negligible proportions.

The same type of approximation for the zero order mode term is

$$
\left|\nu(\nu+1) P_{\nu}(-\cos \theta) / 2 \sin \nu \pi\right| \simeq \nu(\nu+1) \cos [(\nu+(1 / 2))(\pi-\theta)-(\pi / 4)] /(\sin \nu \pi) \sqrt{(2 \nu+1) \pi \sin \theta}
$$

for $n=0$

$$
\text { if }|\nu| \theta \gg 1 \text { and }|\nu|(\pi-\theta) \gg 1 \text {. }
$$

The degree $\nu(0)$ has close to unit value.

By comparison to the higher order mode (see (10)) the zero order mode predominates at any large distance. Even its nulls are blunted by the slight propagation losses of the zero order mode.

As the distance (polar angle) is reduced, the logarithmic increase of $P_{\nu}(-\cos \theta)$ (see (4)) eventually comes into play where all modes become important. In order for the zero order mode to predominate it is required that $|\nu(n)| \theta>>1$ be true for $n \geqslant 1$ so that the polar angle will stay well outside the range of logarithmic variation for high order modes.

The distance at which a particluar higher order mode can be neglected is given by

$$
\theta a>>a /|\nu(n)| \simeq h / \pi n \text { for } n \geqslant 1((8) \text { used for }|\nu|) .
$$

Obviously the first order mode is the limiting case. The minimum distance is simply required to be large compared to the height of the ionosphere. (As in ordinary waveguides the modes below cutoff are negligible a few guide widths downstream.) A distance of $500 \mathrm{~km}$ satisfies this last requirement under either day or night conditions. Note that this result is independent of frequency (to the first order) over the range of $5 \mathrm{~Hz}$ to $1 \mathrm{kHz}$.

An example of the relative magnitude of the zero order and the first order modes is shown in figure 1 . The first two terms in the series for the normalized vertical field, $E_{r} /\left(\eta I d s / 2 k h a^{2}\right)$, are shown there. The zero order mode $(n=0)$ is shown in approximate form over the three ranges of distance that were outlined previously. The frequency of propagation is about $11 \mathrm{~Hz}$, which

FigURE 1. Magnitude of the zero order and first order mode terms from the series for normalized vertical electric field versus distance from the source, using approximations from the text.

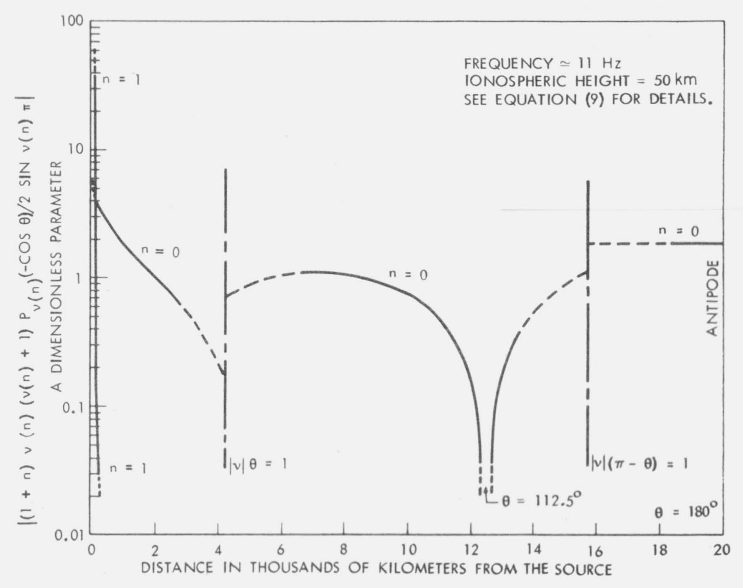


corresponds to a minimum in the natural resonance structure. The height of the ionosphere is $50 \mathrm{~km}$ as might be appropriate during the daytime. More will be said about the behavior of the zero order mode (with distance from the source) in later parts of this paper. But note that the first order mode has no important relative magnitude until the distance from the source is below $200 \mathrm{~km}$. The selection of $500 \mathrm{~km}$ as the minimum distance for predominance of the zero order mode is very conservative.

\subsection{Bounding Distances Between the Three Approximations for the Zero Order Mode}

Figure 1 demonstrates the point that for low frequencies there is a range of source distances, from $500 \mathrm{~km}$ out to the place where $|\nu| \theta=1$, over which the normal asymptotic form for $P_{\nu}(-\cos \theta)$ does not apply. (The approximations of sec. 2 have been used in plotting the three ranges of distance shown for the zero order mode.) The equivalent distance from the source that lies between these two ranges of approximation, is equal to

$$
\theta a=a /|\nu(0)|=a /\left(-(1 / 2)+\sqrt{\left(k a S_{0}\right)^{2}+(1 / 4)}\right)
$$

where use has been made of (5). Figure 2 is a plot of this boundary distance from the source as a function of propagation frequency. If the distance is measured instead from the antipode of the source then the same curve is a second boundary between a second pair of distance ranges based on $|\nu|(\pi-\theta)=1$ (see fig. 1). Note that at higher frequencies this boundary is closely given by one-sixth wavelength.

It should be emphasized that the necessity for three different types of approximation to $P_{\nu}(-\cos \theta)$ really only arises at the lower end of the ELF band in the normal propagation analysis. Figure 2 shows that the range of $5 \mathrm{~Hz}$ to $100 \mathrm{~Hz}$ is the frequency band affected. When the frequency exceeds $100 \mathrm{~Hz}$ the boundary distance is less than $500 \mathrm{~km}$. The boundary distance is exceeded before the $500 \mathrm{~km}$ point is reached at which the zero order mode predominates. Thus, it can be ignored in the normal propagation analysis that assumes solely zero order mode. Strictly speaking the special approximation for the area around the antipode of the source still applies. However, one might argue that the area involved is small enough for frequencies above $100 \mathrm{~Hz}$ that it could be ignored for all practical purposes.

Near the low end of the ELF band at $5 \mathrm{~Hz}$ the curve of figure 2 shows another phenomenon. The boundary distance between approximation regions has reached one-quarter of the distance around the earth. This boundary applies both around the source and its antipode. This means that the middle range of distances has disappeared, and the normal asymptotic expansion for $P_{\nu}(-\cos \theta)$ does not apply at any distance.

\subsection{Higher Order Modes Within $500 \mathrm{~km}$ of the Antipode of the Source}

One point of rigor remains in the treatment presented here. The antipode of the source is a natural focus at which the various mode amplitudes can be expected to increase. The zero

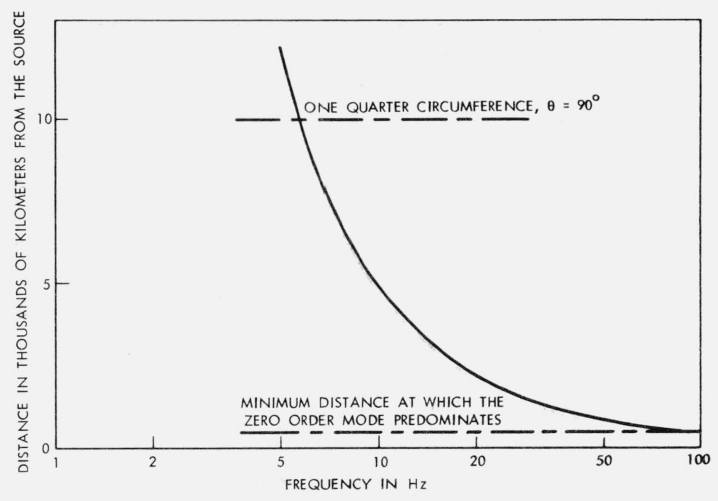

FIGURE 2. Distance from the source that separates two ranges of approximation for the zero order mode.

(Same curve is the boundary between another pair of ranges where distance is from the antipode of the source.) 
order mode should be checked to see that it predominates here also. Equation (10) shows the magnitude of the higher order modes would grow without bound except that that result does not apply within $500 \mathrm{~km}$ of the antipode. (An argument similar to that of sec. 3.1, but using $|\nu(n)|(\pi-\theta)>>1$ instead of $|\nu(n)| \theta>>1$, shows that (10) does not apply within $500 \mathrm{~km}$ of the antipode as well as $500 \mathrm{~km}$ of the source.) The correct approximation for high order mode terms within $500 \mathrm{~km}$ of the antipode is

$$
\begin{aligned}
& \left|\nu(\nu+1) P_{\nu}(-\cos \theta) / \sin \nu \pi\right| \simeq 2(\pi a n / h)^{2} \exp (-(\pi a n / h) \pi) \\
& \text { for } n \geqslant 1 \\
& \text { if }|\nu|(\pi-\theta)<<1 \text { (see sec. } 2 \text { for approximations). }
\end{aligned}
$$

The $\theta$ dependence has disappeared as the mode amplitude approaches a constant. The net conclusion is that even around the antipode the higher order mode terms $(n \geqslant 1)$ are negligible compared to the zero order mode. The exponential factor of (14) insures this fact just as in (10). The large differential attenuation between the higher order and zero order modes is not overcome by the focusing experienced at the source antipode.

\section{ELF Noise Spectrum Extensions}

The integral for ELF noise power spectrum from a vertical stub antenna, due to lightning discharge, has already been derived [Galejs, 1961a]. The section evaluates this integral expression when the sources are near the antenna or its antipode and the low-frequency end of the ELF band ( 5 to $100 \mathrm{~Hz}$ ) is being considered. For the readers convenience several parameters are redefined in this section. A similar discussion, appropriate to the vertical electric field intensity has been given by Wait [1960b].

\subsection{Integral Expression}

The starting point is the integral, just referred to, for received noise power spectrum of a short vertical antenna.

$$
\begin{gathered}
G(i \omega)=g(i \omega) \frac{2 \pi \nu(\nu+1) \sigma(\sigma+1)}{16 k^{2} a^{2} h^{2} \sin \nu \sigma \sin \sigma \pi} \\
\int_{\theta_{1}}^{\theta_{2}} P_{\nu}(-\cos \theta) P_{\sigma}(-\cos \theta) \sin \theta d \theta
\end{gathered}
$$

where $g(i \omega)=$ quantity proportional to the squared dipole moment of the lightning discharges per unit area (assumed independent of position), and

$\omega=$ radian frequency,

$k=$ free space, plane wave propagation factor,

$a=$ radius of the earth,

$h=$ height of the lower edge of the ionosphere,

$\theta=$ polar angle at the center of the earth measured from noise source to receiving antenna,

$S_{0}=$ zero order root of the modal equation, a complex number that is largely real and close to unity.

$\nu(\nu+1)=\left(k a S_{0}\right)^{2}$,

$\sigma=$ complex conjugate of $\nu$,

$P_{\nu}(z)=$ Legendre function of $z$ with degree $\nu$. 
This expression assumes a constant surface density excitation of the earth-ionosphere cavity over a belt bounded by the two polar angles, $\theta_{1}$ and $\theta_{2}$. Variations of surface excitation are accommodated by a piecemeal application of (15). Keep in mind that the radio-propagation depends only on polar angle and not azimuth angle.

In this paper approximations will be made after integration so that all three possible ranges of approximation can be handled with one integration. According to Bateman Manuscript Project [1953] the integral equals

$$
\begin{aligned}
\int_{\theta_{1}}^{\theta_{2}} P_{\nu}(-\cos \theta) P_{\sigma}(-\cos \theta) \sin \theta d \theta & \\
= & {\left[\frac{\sin \theta\left(P_{\sigma}(-\cos \theta) P_{\nu}^{1}(-\cos \theta)-P_{\nu}(-\cos \theta) P_{\sigma}^{1}(-\cos \theta)\right)}{(\nu-\sigma)(\nu+\sigma+1)}\right]_{\theta_{1}}^{\theta_{2}} }
\end{aligned}
$$

There does not seem to be any simple reduction of this form. So the next step is to approximate the various Legendre functions to obtain a result in convenient form for numerical computation.

\subsection{Approximate Results}

Consider first the case when the polar angle $\theta$ lies in the middle range. Using the asymptotic forms of (2), combined with (15) and (16), the noise power spectrum is approximated by

$$
\begin{gathered}
G(i \omega) \simeq g(i \omega) \frac{|\nu(\nu+1)|^{2}}{4(k a h)^{2}|\nu+(1 / 2)|(\cos (\nu-\sigma) \pi-\cos (\nu+\sigma) \pi)} \\
\cdot\left[\frac{\cos (\nu+\sigma+1)(\pi-\theta)}{(\nu+\sigma+1)}-\frac{\sin (\nu-\sigma)(\pi-\theta)}{(\nu-\sigma)}\right]_{\theta_{1}}^{\theta_{2}} \\
\text { for }|\nu| \theta>>1 \text { and }|\nu|(\pi-\theta)>>1 .
\end{gathered}
$$

This is equivalent to the answer obtained previously by Galejs [196la]. It has already been noted that the asymptotic forms used here for $P_{\nu}$ and $P_{\nu}^{1}$ break down when the source is close to the antenna or its antipode.

When the noise sources are near the antipode of the antenna then the approximations of (3) are used with (15) and (16) to get

$$
G(i \omega) \simeq g(i \omega) \frac{\pi|\nu(\nu+1)|^{2}}{4(k a h)^{2}(\cos (\nu-\sigma) \pi-\cos (\nu+\sigma) \pi)} \cdot[-\sin \theta \cos \theta / 2]_{\theta_{1}}^{\theta_{2}} \text { for }|\nu|(\pi-\theta)<<1 .
$$

The noise power observed drops to zero as the source approaches the antipode due in part to the vanishing circumference of the belt being integrated. (This is contrary to the middle range expression that is finite at the antipode.)

The remaining condition is when the sources are near the antenna, itself. (But still farther than $500 \mathrm{~km}$ away so that only the zero order mode is present.) Then the approximations of (4) are used with (15) and (16) to obtain

$$
\begin{aligned}
& G(i \omega) \simeq g(i \omega) \frac{|\nu(\nu+1)|^{2}}{2(k a h)^{2}(\nu-\sigma)(\nu+\sigma+1)} \\
& \cdot\left\{-\frac{\sin (\nu-\sigma) \pi}{(\cos (\nu-\sigma) \pi-\cos (\nu+\sigma) \pi)}+\frac{(\nu-\sigma)}{\pi} \sum_{n=0}^{\infty} \frac{1}{|\nu+1+n|^{2}}\right\} \cdot[\cos \theta / 2]_{\theta_{1}}^{\theta_{2}}
\end{aligned}
$$

for $|\nu| \theta \ll 1$. 
FIGURE 3. Magnitude of ratio error squared from using midrange approximation, near the antenna or its antipode, for the electric field intensity.

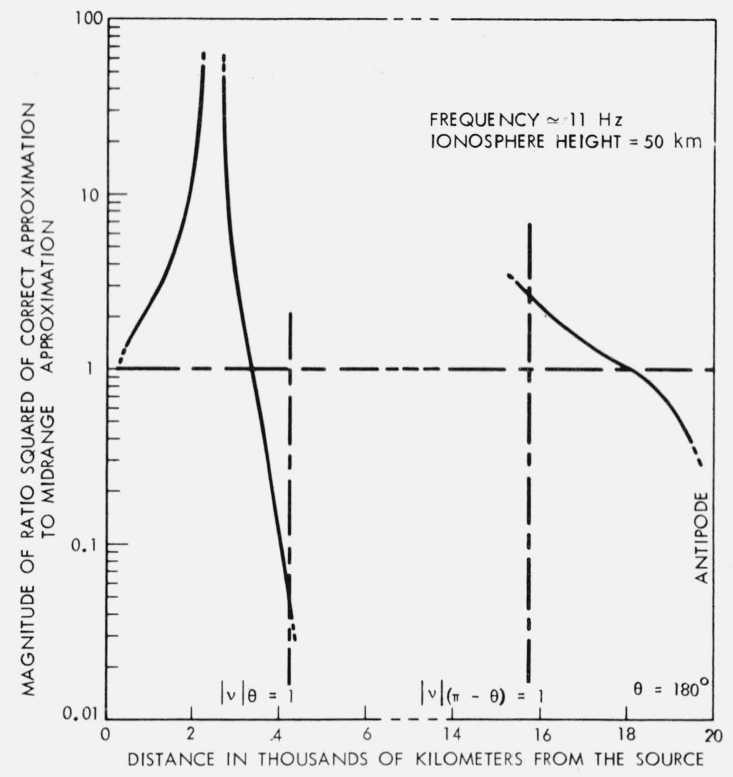

Thus, when the sources are near the antenna itself the contribution to the observed noise power spectrum still remains finite. This is again because the vanishing circumference of the belt being integrated tends to dominate the process.

These three results for noise power spectrum [(17), (18), and (19)] cover all the possibilities. It may be that the limits of integration are in different regions of approximation. Since the exact integration is being approximated such a mixture of limits is easy to handle. Each limit is used in the appropriate approximation and the two subtracted to get the total answer.

In figure 3 plots are presented to show the large error near the antenna or its antipode in calculating noise spectrum contributions using only the midrange approximation. The same parameters are used in figure 1; frequency is about $11 \mathrm{~Hz}$ and ionospheric height equals $50 \mathrm{~km}$. What is plotted in figure 3 is essentially the ratio of noise power contributions from an elementary sized area as approximated these two different ways.

\section{Discussion and Conclusions}

It has been shown that for terrestrial radio wave propagation in the middle ELF band (frequencies of $5 \mathrm{~Hz}$ to $1 \mathrm{kHz}$ ) the zero order waveguide mode is predominant when the distance from the source exceeds roughly $500 \mathrm{~km}$. The theory of noise power spectrum in the ELF band that is extended in this paper assumes a "locally quiet" receiving site. In the sense employed here the phrase "locally quiet" means there are no thunderstorms within $500 \mathrm{~km}$. Only the zero order modes propagating from lightning strokes need be accounted for.

It has also been shown how the theoretical terrestrial ELF noise spectrum has different approximate forms depending on whether the source is close to the antenna, or its antipode, or neither. This last modification of theory is chiefly important for frequencies below $100 \mathrm{~Hz}$. The critical distances from the source or its antipode are roughly given by one-sixth wavelength.

It is interesting to look at plots by Wait [1960b] of $\log \left(\left|E_{z}\right| \sqrt{\rho}\right)$ versus $(\rho)$ for frequencies down to $50 \mathrm{~Hz}$. (See fig. 1 of that reference; $E_{z}$ is the vertical electric field and $\rho$ is the distance from the noise source or lightning stroke.) At $50 \mathrm{~Hz}$ the curve is linear clear down to a distance from the source of less than $400 \mathrm{~km}$. Presumably this is due to the predominance of the zero order mode at larger distances. This plot at $50 \mathrm{~Hz}$ shows that Wait's restriction of one-sixth wavelength for the limiting distance for linearity is more stringent than necessary. (At $50 \mathrm{~Hz}$, a distance of one-sixth wavelength equals $1000 \mathrm{~km}$.) 
The zero electric field at $\theta=112.5^{\circ}$ in figure 1 is a result predicted by the same reasoning that accounts for the ELF resonance and antiresonance frequencies. As Wait [1964] has observed the resonance frequencies are closely given by

$$
\left(k\left|S_{0}\right|\right)(2 \pi a)-\pi \simeq 2 \pi n
$$

where $k=$ free space, plane wave propagation factor

$S_{0}=$ zero order root of the modal equation

$a=$ radius of the earth

$n=1,2,3$. . .

The explanation is that resonance occurs when the magnitude of phase retardation for one circuit around the world is equal to an integer number of complete cycles. The subtraction of $\pi$ accounts for separate $\pi / 2$ phase advances that occur at the caustic points of each source and its antipode. Similarly the antiresonance points occur when

$$
\left(k\left|S_{0}\right|\right)(2 \pi a)-\pi \simeq 2 \pi n-\pi
$$

so that a complete circuit tends toward phase cancellation.

Following the same train of thought there should be minimum electric field intensity at an angle from the source $\theta$ where

$$
\left[\left(k\left|S_{0}\right|\right)((2 \pi-\theta) a)-(3 \pi / 4)\right]-\left[\left(k S_{0}\right)(\theta a)-(\pi / 4)\right] \simeq \pi(2 n+1) .
$$

The difference in phase retardations equals a number of complete cycles plus one-half. In the example plotted in figure 1 the frequency of $11 \mathrm{~Hz}$ is an antiresonant point where $k\left|S_{0}\right| a \simeq 2$. Substituting this in (22) and solving gives $\theta \simeq 112.5^{\circ}$ as the only angle in the middle range where phase cancellation tends to occur. The graph verifies this conclusion.

The extensions of the formulas for terrestrial noise power spectrum given in this paper will have application in a future paper concerned with accounting for temporal and geographical distribution of lightning strokes.

This work was sponsored by the Department of the Navy, Office of Naval Research, Washington, D.C.

The author acknowledges several helpful discussions with R. V. Row of this Laboratory during the course of the work.

\section{References}

Balser, M., and C. A. Wagner (1960), Observations of earth-ionosphere cavity resonances, Nature 188, 638-641.

Balser, M., and C. A. Wagner (1962), Diurnal power variations of the earth-ionosphere cavity modes and their relationship to worldwide thunderstorm activity, J. Geophys. Res. 67, No. 2, 619-625.

Bateman Manuscript Project (1953), Higher transcendental function, 1, ch. 3 (McGraw-Hill Book Co., Inc., New York, N.Y.). Galejs, J. (196la), Terrestrial extremely low frequency noise spectrum in the presence of exponential ionospheric conductivity profiles, J. Geophys. Res. 66, No. 9, 2787-2792.

Galejs, J. (1961b), ELF waves in the presence of exponential ionospheric conductivity profiles, IRE Trans. Ant. Prop. AP-9, No. 6, 554-562.

Galejs, J. (1962), A further note on terrestrial extremely low-frequency propagation in the presence of an isotropic ionosphere with an exponential conductivity-height profile, J. Geophys. Res. 67, No. 7, 2715-2728.

Magnus, W., and F. Oberhettinger (1949), Formulas and theorems for the special functions of mathematical physics, ch. 4 (Chelsea Publ. Co., New York, N.Y.).

Raemer, H. R. (1961), On the spectrum of terrestrial radio noise at extremely low frequencies, J. Res. NBS 65D (Radio Prop.), No. 6, 581-593. 
Schumann, W. O. (1957), Elektrische Eigenschwingungen des Hohlraumes Erde-Luft-Ionosphere, Z. angew, Phys. 9, 373-378.

Wait, J. R. (1960a), Terrestrial propagation of very-low frequency radio waves, J. Res. NBS 64D (Radio Prop.), No. 2, 153-204.

Wait, J. R. (1960b), Mode theory and the propagation of ELF radio waves, J. Res. NBS 64D (Radio Prop.), No. 4, 387-404.

Wait, J. R. (1964), On the theory of Schumann resonances in the earth-ionosphere cavity, Can. J. Phys. 42, No. 4, 575-582.

(Paper 69D7-532) 